\title{
Dynamic simulation of sheared suspensions. I. General method
}

\author{
Georges Bossis \\ Laboratoire de Physique de la Matière Condensèe, Universitè de Nice, Parc Valrose, 06034 Nice Cedex, France \\ John F. Brady \\ Department of Chemical Engineering, Massachusetts Institute of Technology, Cambridge, Massachusetts \\ 02139
}

(Received 31 October 1983; accepted 12 December 1983)

\begin{abstract}
A general method is presented for simulating the dynamical behavior of a suspension of particles which interact through both hydrodynamic and nonhydrodynamic forces. In the moleculardynamics-like simulation there are two different procedures for computing the interactions among particles: a pairwise additivity of forces and a pairwise additivity of velocities. The pairwise additivity of forces is the preferred method as it preserves the hydrodynamic lubrication forces which prevent particles from overlapping. The two methods are compared in a simulation of a monolayer of identical rigid non-Brownian spherical particles in a simple shear flow. Periodic boundary conditions are used to model an infinite suspension. Both methods predict the presence of a shear induced anisotropic local structure whose form and strength depend on the concentration of particles, the nonhydrodynamic forces, and the shear rate. Increasing the particle concentration up to near the maximum fraction that can still flow results in a transition to a layered structure in which planes of particles slide relative to one another. The anisotropic local structure and transition to a layered structure predict a non-Newtonian suspension rheology.
\end{abstract}

\section{INTRODUCTION}

Interest in suspension mechanics has experienced a marked increase in recent years, with contributions coming from many different fields, e.g., engineering, chemistry, physics, and mathematics. The basic problem is to predict the macroscopic transport properties of a suspension-electrical or thermal conductivity, viscosity, sedimentation rate, etc.-from the microstructural mechanics, i.e., from the interactions among the suspended particles and from their distribution in space. The articles by Batchelor, ${ }^{1}$ Brenner, ${ }^{2}$ Jeffrey and Acrivos, ${ }^{3}$ and Russel ${ }^{4}$ provide a comprehensive review of the theoretical and experimental work in this area. For the most part, theoretical studies have been limited to dilute concentrations at zero particle Reynolds number where single- or two-particle interactions dominate and have sought to determine the macroscopic properties by an expansion in number density or volume fraction. Unfortunately, agreement with experiment is generally found up to only a few volume percent. Nevertheless, these theories have identified the fundamental mechanisms operating in suspensions and provide an important foundation upon which to base further studies.

Extending dilute suspension analyses to higher concentrations poses two problems. The first is the determination of the many-body interactions among particles, particularly, the many-body hydrodynamic interactions. While extremely complex, using various renormalization and diagrammatic-type techniques progress can be, and has been, made in computing many-body hydrodynamic interactions. ${ }^{5,6}$ These calculations are by no means exact for all particle-particle separations, but they do give some indication of the importance of three-body and higher-order effects. ${ }^{7}$

The second problem is the determination of the spatial distribution of particles-the configuration of the microstructure. To date, no progress has been made beyond the dilute two-particle limit. This is due in large part to the fact that in suspensions in which particles undergo relative motion, as in response to an externally imposed driving force (bulk shear flow e.g.), the configuration cannot be specified $a$ priori, but must be found as part of the problem. This aspect sets the viscosity and sedimentation problems apart from the conductivity ones, for in the latter the distribution of particles can be supposed known or given at the outset. Indeed, the configuration of the microstructure in a flowing suspension is a dynamic rather than static entity. The dilute suspension theories have all assumed or calculated stationary configurations and are therefore applicable only to the time averaged behavior. Our principle interest in this work lies in the dynamic and configurational aspects of suspensions.

The only rigorous analyses of concentrated suspensions have assumed the particles are distributed in a regular array or lattice. ${ }^{8-12}$ Although the many-body interaction problem can now be solved exactly, the assumption of perfect regularity is highly restrictive and is at best only an approximation for flowing suspensions.

These notions and problems are quite familiar to statistical mechanicians who deal with the equilibrium and transport properties of dense gases and liquids, and indeed many analogies can be made. Molecular dynamics has proved an effective means for determining the configuration of particles, in particular, the pair distribution function $g(\mathbf{r})$, from which most properties of dense systems can be calculated. With the recent advances in the hydrodynamical aspects of suspensions, it seems appropriate to try to apply some of the molecular-dynamics ideas to suspensions. We are not aware of any study which has attempted a molecular-dynamicslike simulation of sheared suspensions, although several papers have appeared on Brownian dynamics with hydrodynamic interactions which has some features in common with the present problem. ${ }^{13,14}$ It is the purpose of this paper to present a general method for performing such simulations 
and to present results for some model suspensions.

In Sec. II we shall outline a method for simulating the dynamic behavior of a suspension of rigid, non-Brownian particles subjected to a bulk, macroscopic, shear flow when the particle Reynolds number is small. The particles are allowed to interact both hydrodynamically and through interparticle forces, the form of which is arbitrary. A procedure is also presented for overcoming the convergence difficulties associated with the long-range hydrodynamic interactions between particles-the velocity disturbance for a force-free particle decays as $1 / r^{2}$-allowing one to simulate a system with periodic boundary conditions. As has been done in most molecular dynamics calculations, in order to reduce computation time, pairwise additivity of the interactions is assumed. It will be seen below that pairwise additivity of hydrodynamic interactions can be carried out in two, very different, ways. The first method entails superposing velocity disturbances, or said differently using pairwise additivity in the so-called mobility matrix. The second method is a superposition of forces rather than velocity disturbances, or a pairwise additivity in the resistance matrix. Although it is clear that these two methods are not the same, there has been no study which has examined the predictions of one over the other, and a major component of this work is to carry out such a comparison. We shall show that the superposition of forces follows more closely the proper physics in that the hydrodynamic lubrication forces that prevent particles from touching are preserved. These lubrication forces are not preserved with the superposition of velocities and particles can freely overlap unless strongly repulsive interparticle forces are present. It will also be seen that the superposition of forces includes more than a strict pairwise additivity and in some sense many-body interactions are taken into account. Hence, it is the preferred method. The Brownian-dynamics simulations referred to previously all used a superposition of velocities.

Following the general method, in Sec. III we shall apply this technique to the simulation of a monolayer of identical rigid spherical particles freely suspended in a simple shear flow. In the monolayer all particles centers lie in the plane of shear (cf. Fig. 2), reducing the number of degrees of freedom for each sphere from six to three with an obvious saving in computation time. While an idealization, a monolayer is a realizable suspension flow and experiments have been performed on such systems. ${ }^{15,16}$ More importantly, however, it is felt that the flow induced structure in the plane of shear, as measured by the pair distribution function, should not differ significantly from that in a fully three-dimensional shear flow. In addition to hydrodynamic interactions, the particles also interact through repulsive DLVO-type colloidal forces.

In Sec. IV we present the results of the numerical simulations principally in terms of the pair distribution function $g(\mathbf{r})$. Simulations were performed with both 25 and 100 particles, and the results show that 25 particles yield accurate distribution functions. It will be seen that the shear flow induces a pronounced anisotropic local structure which has in general both radial and angular dependence. The precise form of this structure depends on the concentration of particles, the interparticle force, and the shear rate. The angular structure that develops can be traced to the repulsive interparticle force-it is absent when there is no such forcewhile the radial structure is primarily an excluded volume effect. The local structures found by both simulation methods agree qualitatively, but there are quantitative differences. It will also be seen that increasing the particle density up to near the maximum areal fraction that can still flow results in a transition to an ordered layered structure.

We do not discuss in any depth the macroscopic transport properties that can be determined from the dynamic simulations, as the purpose of this paper is to demonstrate the method and indicate the types of behavior to be expected in sheared suspensions. We can say, however, that the anisotropic local structure that is induced by the shear flow gives rise to non-Newtonian rheological behavior - the fluid cannot be characterized by a simple scalar viscosity. Subsequent papers will give results for the bulk rheological properties as a function of concentrtion, interparticle force, and shear rate. Future work will also show how to extend the present method to include the effects of rigid boundaries and Brownian motion.

\section{SIMULATION METHOD}

We consider solid rigid particles suspended in a Newtonian fluid of viscosity $\mu$ and density $\rho$. The equations of motion for the fluid are the familiar Navier-Stokes equations for an incompressible constant property fluid, while those for the particles can be written in the following matrix form:

$$
\mathbf{M} \cdot \frac{d \mathbf{U}}{d t}=\mathbf{F}_{H}+\mathbf{F}_{P}
$$

In Eq. (1) $\mathrm{U}$ is a column vector of dimension $6 N$ containing the translational and angular velocities of all $N$ particles under consideration. The particle and fluid velocities are related by the no slip condition at the particle surfaces. $\boldsymbol{M}$ is a generalized mass/moment of inertia matrix of dimension $6 N \times 6 N . \mathbf{F}_{H}$ and $\mathbf{F}_{P}$, also of dimension $6 N$, are respectively the hydrodynamic and nonhydrodynamic force-torque vectors. When an appropriately defined particle Reynolds number is small (see below) and in the absence of Brownian motion, i.e., an appropriately defined Péclet number is large (see below), the particle inertia may be neglected in comparison with the hydrodynamic and nonhydrodynamic force torques. Then, Eq. (1) becomes simply

$$
\mathbf{F}_{H}+\mathbf{F}_{P}=0 \text {. }
$$

As shown by Brenner and co-workers, ${ }^{17,18,10}$ because of the linearity of the governing Stokes equations for the inertia-free particle motion, the hydrodynamic force-torque vector for particles suspended in a fluid undergoing a bulk linear shear flow characterized by the constant traceless (from incompressibility) symmetric rate of strain tensor $E$ and constant vorticity $\omega$ can be written as

$$
\mathbf{F}_{H}=\mathbf{R} \cdot \mathbf{U}^{*}+\boldsymbol{\Phi}: \mathbf{E}
$$

In Eq. (3) $U^{*}$ is the particle translational-angular velocity relative to the bulk fluid translational-angular velocity evaluated at the particle's center. For example, suppose particle $\alpha$ is located at the point $\mathbf{X}_{\alpha}$ relative to the origin for the bulk 
flow. The fluid's bulk translational velocity evaluated at the particle center $\mathbf{X}_{\alpha}$ is then $\mathbf{E} \cdot \mathbf{X}_{\alpha}$. The bulk angular velocity at the particle center is simply the constant $\omega$. Hence, for particle $\alpha$ its translational velocity $\mathbf{u}_{\alpha}$ is given by

$$
\mathbf{u}_{\alpha}=\mathbf{u}_{\alpha}^{*}+\mathbf{E} \cdot \mathbf{X}_{\alpha}
$$

and its angular velocity about its center $\mathbf{X}_{\alpha}$ is

$$
\mathbf{\Omega}_{\alpha}=\mathbf{\Omega}^{*}+\boldsymbol{\omega} \text {. }
$$

The translational-angular velocity for particle $\alpha, \mathbf{U}_{\alpha}$, is composed of the above as $\mathbf{U}_{\alpha}=\left(\mathbf{u}_{\alpha}, \Omega_{\alpha}\right)$. For the case of steady simple shear flow with shear rate $\gamma$ in the $(x, y)$ plane, the bulk rate of strain tensor and vorticity are given by

$$
\mathbf{E}=\frac{\dot{\gamma}}{2}\left(\begin{array}{lll}
0 & 1 & 0 \\
1 & 0 & 0 \\
0 & 0 & 0
\end{array}\right)
$$

and

$$
\omega=-\frac{\dot{\gamma}}{2}(0,0,1)
$$

We have used the word "bulk" in characterizing the linear shear flow to indicate that $E$ ad $\omega$ are constant (in space, although they may vary in time) macroscopic quantities. How these relate to the actual mechanism causing the homogeneous shear flow will be discussed below when we consider the periodic boundary conditions. For the moment, we are simply interested in the structure of the hydrodynamic force torque as given by Eq. (3).

The matrix $R$ in Eq. (3) is known as the grand resistance matrix and is a purely geometric quantity that depends on the instantaneous configuration-relative position, orientation, etc. - of all $N$ particles. The $6 N \times 6 N$ matrix $R$ relates the force torque exerted by the suspending fluid on each particle due to the translational-angular motion of all $N$ particles (including the designated one) relative to the fluid. The $6 N \times 3 \times 3$ matrix $\Phi$ is known as the shear resistance matrix and it too is a purely geometric, configuration dependent entity. $\Phi$ gives the force torque on a particle in the suspension due to the bulk shear flow. Both $R$ and $\Phi$ possess many symmetry properties resulting from the Lorentz reciprocal theorem for Stokes flow. ${ }^{10,17-20}$ More details of the structure of $R$ and $\Phi$ will be given below when we discuss the pairwise additivity assumption. The inverse of $R$ is known as the mobility matrix.

We have so far left the nonhydrodynamic force $F_{p}$ unspecified. $F_{p}$ stands for any interparticle forces that might be present, e.g., colloidal forces, and for any externally imposed forces such as those arising from gravitational and electrical fields. In general, $\mathbf{F}_{p}$ will also depend on the instantaneous configuration of particles.

In the linear shear flow where $\dot{\gamma}^{-1}$ sets the time scale, the condition for neglect of particle inertia is that the particle Reynolds number $\operatorname{Re}=\rho\left(\gamma a^{2} / \mu\right)$ be much less than unity. Here $a$ is a characteristic size of the particles. In order that Brownian motion be neglected, the particle Péclet number $\mathrm{Pe}=6 \pi \mu a \dot{\gamma} / k T$ must be much larger than unity. Here, $T$ is the absolute temperature, $k$ is Boltzmann's constant, and we have made use of the Stokes-Einstein relation for the particle diffusivity $D=k T / 6 \pi \mu a$. For water at room temperature, spherical particles in excess of $5 \mu \mathrm{m}$ in diameter have
Péclet number of $0(100)$ for shear rates of $0\left(1 s^{-1}\right)$. Under the same conditions, the particle's Reynolds number is $0\left(10^{-5}\right)$. Thus, only for very small prticles or at very low shear rates is Brownian motion important. If the particle motion were determined by an external force rather than the shear flow, this force would set the characteristic velocity from which to estimate the particle Reynolds and Péclet numbers. Note, this limitation on the particle Reynolds number does not mean that the Reynolds number for the bulk motion must be small.

To simulate the dynamics of a suspension we determine the particle velocities from Eq. (2), along with Eq. (3) for the hydrodynamic force torques and whatever nonhydrodynamic force torques $\mathbf{F}_{p}$ are appropriate, i.e.,

$$
\mathbf{R} \cdot \mathbf{U}^{*}+\boldsymbol{\Phi} \cdot \mathbf{E}+\mathbf{F}_{p}=0 \text {. }
$$

Or, solving for the velocities

$$
\mathbf{U}^{*}=-\mathbf{R}^{-1} \cdot\left\{\boldsymbol{\Phi}: \mathbf{E}+\mathbf{F}_{P}\right\},
$$

where $\mathbf{R}^{-1}$ is the inverse of the matrix $R$. Equation (5) is deceptively simple because $R, \boldsymbol{\Phi}$ and $\mathbf{F}_{P}$ are all configuration dependent and thus implicit functions of time. Having found the velocities at a given instant, the new particle positions can be found a short time $\Delta t$ later, but then the configuration will have changed, and $R, \Phi$, and $F_{P}$ must be recomputed before the particle trajectories can be continued. We shall present a scheme for doing this in the next section, but first we must address two important questions: periodic boundary conditions and pairwise additivity. Note that by neglecting the particle inertia, we have first order differential equations to solve for the particle positions rather than second order as is customary in molecular dynamics.

In order to simulate a suspension of "infinite" extent, periodic boundary conditions are needed to restrict the number of particles whose motion is followed. Figure 1 shows a periodic box of length $L$ and height $H$ for simple shear flow in the $(x, y)$ plane. (We shall only consider simple shear flow in this paper; different conditions may be needed in other

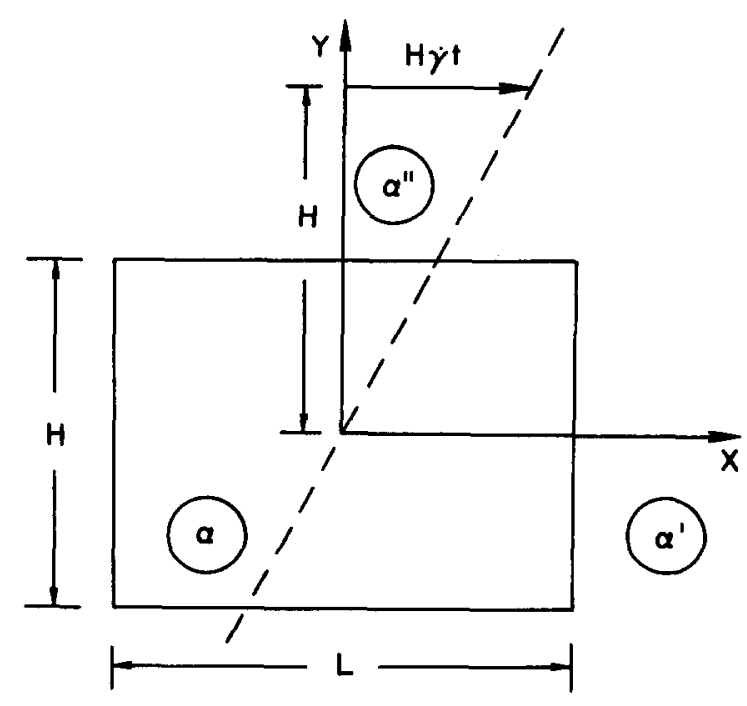

FIG. 1. Diagram of the periodic boundary conditions showing the location of the images $\alpha^{\prime}$ and $\alpha^{\prime \prime}$ in the $x$ and $y$ directions. The image $\alpha^{\prime \prime}$ is translated in $x$ by the amount $\boldsymbol{H} \dot{\gamma}$. This preserves the shear flow in the $y$ direction. 
situations.) The image $\alpha^{\prime}$ of particle $\alpha$ outside the box in the $x$ or $z$ direction is just the usual translation by $L$. In the $y$ direction, however, a particle which leaves the top of the box does not enter the botom at the same $x$ coordinate. As shown by Evans, ${ }^{21}$ the image $\alpha$ " of particle $\alpha$ in the $y$ direction is translated as illustrated in Fig. 1 by an amount $H \dot{\gamma} t$ where $t$ is time. This translation reproduces the shear flow in the $y$ direction.

By periodic boundary conditions we represent an infinite suspension as a spatially periodic array of identical cells. It is easy to see that for such an array the rate of strain $E$ and vorticity $\omega$ used in deriving Eq. (3) are indeed well defined macroscopic constants. In fact, this has been proven by Adler, et $a l .^{10}$ Thus, simple shear flow with periodic boundary conditions is well posed and is intended to model the behavior of a suspension in regions far removed from the boundaries creating the simple shear. To include boundary effects the formulation in Eq. (3) cannot be used because a bulk linear shear flow does not necessarily exist. For this case a well posed problem is one in which the boundaries move with prescribed velocities rather than imposing an average shear rate. In a subsequent paper we shall show how to incorporate boundary effects in the simulation method.

In using periodic boundary conditions, we assume that a particle in the center of its periodic cell interacts only with other particles that are also within the cell. Interactions with particles outside the cell are neglected, and this step must be justified because the hydrodynamic interactions between particles are very long ranged. For force-free particles in a linear shear flow the velocity disturbance due to a particle decays as $1 / r^{2}$. (The particles act like force dipoles.) To show that we can neglect the particles outside the periodic cell when determining the trajectory of a given particle, we appeal to the work of O'Brien. ${ }^{22}$ By using Green's theorem for Stokes flow one can show, as O'Brien has for Laplace's equation, that force-free particles outside a volume of characteristic radius $R$ in total contribute zero to the velocity of a particle at the center of this volume with an error $0\left(1 / R^{2}\right)$. Thus, as the volume of the periodic cell increases $(R \rightarrow \infty)$, the effect of the particles outside cell can be made arbitrarily small.

Although this can be proven rigorously, there is also a simple physical explanation. As far as the particle at the center of the periodic cell is concerned, particles outside this volume appear, not as discrete particles, but as continuous distribution of force dipoles whose dipole strength depends on the bulk rate of strain $\mathbf{E}$. This uniform distribution of dipoles gives zero contribution to the translational-angular velocity of the central particle. The principal errors come in representing the discrete distribution of particles as a continuous one and in assuming the dipole strength of the continuous distribution is just its bulk average value. The latter error is at most $0\left(1 / R^{2}\right)$ and the former depends on precisely how fast the discrete distribution approaches the continuous one. Our simulations indicate that this approach is sufficiently fast for the above error estimate to be valid. The above derivation (or argument) was developed for force-free particles, i.e., $\mathbf{F}_{\boldsymbol{p}}=0$, but it can be carried through unchanged in the presence of purely interparticle forces. If an external force such as gravity acted on the particles, a similar argument can be employed to justify the use of periodic boundary conditions.

Having established the particle evolution Eqs. (4) or (5) and shown how to implement the periodic boundary conditions, we now turn to the remaining problem of determining the form of the grand resistance and shear resistance matrices and the assumption of pairwise additivty.

Exact solutions for many-body hydrodynamic problems are not available, and it is only within the last ten years that a complete solution for two arbitrarily sized rigid spheres in a linear shear flow has been developed. 17,18,23-35 As the number of references indicate (and this is surely not an exhaustive set) the two sphere results are scattered throughout many papers. Only very recently have they been recomputed and compiled in a single location by Jeffrey an Onishi. ${ }^{36}$ Thus, for two spheres all elements of the grand resistance and shear resistance matrices are known exactly for all separations of the two spheres. This is possible because the geometry of the two sphere problem is completely determined by a single vector-the relative separation vector. It is not the purpose here to present a long list of the elements in the matrices and their symmetry properties as they are available elsewhere, and in particularly concise form in Jeffrey and Onishi, ${ }^{36}$ but some brief comments about the important properties of these matricies are appropriate.

Although exact results are available, there is no closed form analytic solution for all relative separations of the two spheres. At large separations far-field analytical expressions are known via the method of reflections ${ }^{17}$ as a series in inverse powers of the separation distance. At intermediate separations, tabulated numerical results are available $e^{24,34}$ and at small separations analytical results are available from lubrication theory. ${ }^{33}$ The lubrication results are particularly important because many of the elements of $\mathbf{R}$ and $\Phi$ are singular as the separation distance shrinks to the minimum distance for two touching spheres. For example, the force required to push two spheres together at constant relative velocity is proportional to $1 / h$, where $h$ is the minimum distance between the sphere surfaces. The lubrication force becomes infinite as $h \rightarrow 0$ and prevents from touching. Or viewed alternatively, at constant force the relative velocity diminishes proportionally with $h$. The boundaries between these different forms for the matrix elements do not, of course, occur at precise separation distances, but it is necessary to use all three, particularly the lubrication forms, to obtain accurate particle trajectories. Similar exact twosphere results are available for the mobility matrix $\mathbf{R}^{-1}$. Three- and $n$-body effects that have been determined have all been in the form of far-field expansions and have little influence on the singular behavior at small separations.

As discussed in the Introduction, there are two different ways in which to add particle interactions in a pairwise fashion: in the resistance matrix $\mathbf{R}$ or in the mobility matrix $\mathbf{R}^{-1}$. Since the ultimate goal is to determine the particle velocities, $R^{-1}$ must be computed in going from Eq. (4) to Eq. (5) and there is an obvious saving if $\mathbf{R}^{-1}$ can be determined explicitly. Pairwise additivity in the resistance matrix corresponds to a superposition of forces, while in the mobility 
matrix it is a superposition of velocities [cf. Eq. (5)]. It should be clear that these two methods are not the same. Superimposing velocities may be considered a strict pairwise additivity in that the interaction between any two particles $\alpha$ and $\beta$ is the same as if they were alone in the fluid. Interactions with a third particle $\gamma$ are carried out as if the pairs $\alpha-\gamma$ and $\beta-\gamma$ were alone in the fluid, and so on for all $N$ particles. This method of superposition may, and in general does, lead to relative velocities between $\alpha$ and $\beta$ which do not vanish as their separation becomes small, i.e., as $h \rightarrow 0$. Thus, particles can (and do) freely overlap unless there are strong repulsive interparticle forces. Pairwise additivity in the mobility matrix cannot be used at all to simulate a suspension if there are no repulsive interparticle forces, and its use with repulsive forces is debatable since it misses some essential physics.

Superimposing forces by pairwise additivity in the resistance matrix is quite different. Rather than considering pairs of particles alone in the fluid, when the matrix $R$ is inverted to obtain the velocities, all particles are considered simultaneously. Thus, many-body interactions are included in finding the particle trajectories by superposition of forces. The singularities in $\mathbf{R}$ due to the lubrication forces are maintained in the method and particles do not overlap. ${ }^{37}$ This method follows much more closely the proper physics and is to be preferred. This is unfortunate, however, because the computational times associated with solving anew the simultaneous equations embodied in Eq. (5) at each time step greatly exceed those required from the superposition of velocities. We shall present a comparison of the two methods in Sec. IV. In both methods, the same pairwise additivity is used in the shear resistance matrix $\boldsymbol{\Phi}$.

Pairwise additivity in whatever form is an approximation, but one which has proved useful (if not accurate) in conventional molecular dynamics and one we feel will be useful in suspension problems. In dense suspensions, the lubrication forces are the most important and these are modeled correctly by the pairwise superposition of forces. Three-body and higher order effects found from far-field expansions will fade in comparison with the lubrication forces as the separation distances become small. In dilute suspensions most interactions are pairwise, and again the pairwise additivity should be an accurate approximation. Thus, pairwise additivity of forces seems to contain the essential physics. Only by simulation with three-body effects, however, can one truly assess their importance.

Having presented the general method, we can now turn to some of the important details of actually implementing this scheme. We first tested the procedure by computing the trajectories of two identical spheres in the absence of interparticle forces and without the periodic boundary conditions. As discussed previously, asymptotic lubrication formulas were used for nondimensional center-center separations $r$ between 2 and $2.02 ; 2$ is the minimum separation distance. (For some elements of $\mathbf{R}$ the asymptotic formulas were found to be accurate up to 2.1. See Arp and Mason. ${ }^{34}$ ) Tabulated results were used from 2.02 to 3 or 4 depending on the matrix element, and then the farfield results were used for larger separations. The far-field formulas were accurate to at least $0\left(1 / r^{6}\right)$. These results were pieced together from Refs. 17, 18, 24, 33, and 34. The recent compilation by Jeffrey and Onishi ${ }^{36}$ was not available at the time we performed the simulations.

At a given time step the velocities were found by solving the simultaneous linear equation (4) using a standard library routine, and new particle positions were found by stepping forward in time using a fourth order Runge-Kutta scheme. Predictor-corrector methods can also be used for the time stepping, but are not necessary if the time step is sufficiently small. The time step is chosen so that the relative separation does not change significantly in one step; generally, a step size of order $10^{-3}$ (made nondimensional with the shear rate $\gamma$ ) gave accurate results. In this way, we were able to reproduce precisely both the open and closed two-sphere trajectories computed by Batchelor and Green. ${ }^{30}$

Tests were also carried out with more than two spheres. Both open and closed trajectories were found for three, four, and five spheres depending on the initial conditions. In calculations without repulsive colloidal forces, numerical error can cause particles to try to overlap. This can be prevented by reducing the time step, but it becomes very costly. Furthermore, some of the lubrication formulas involve the logarithm of $(r-2)$, and attempting to take the logarithm of a negative number brings the computations to a halt. To overcome this problem, we let particles overlap slightly by fixing the minimum separation in the computation of $\mathbf{R}$ and $\boldsymbol{\Phi}$ at $r=2+10^{-8}$. Note, we are not preventing particles from overlapping, rather we are insuring that the program does not stop executing by taking the logarithm of a negative number. With this procedure, in the absence of interparticle forces, particles would overlap from time to time, but the minimum separation distances found in these tests and in the simulations presented in Sec. IV were never less than 1.99, an overlap of at most $1 \%$, and generally were much beter $\sim 0(1.9999)$. This overlapping is a numerical problem and not due to pairwise additivity-it can occur equally well for two spheres if the time step is not sufficiently small. There is thus a tradeoff between computation time and numerical accuracy as is to be expected. It is also worth noting that separation distances of the order $r-2 \sim 0\left(10^{-8}\right)$ occur quite frequently in trajectory calculations in absence of any repulsive interparticle forces. For millimeter size spheres this implies surface separations the order of Angstroms; clearly, surface roughness and molecular forces may be important, and the validity of the continuum approximation is questionable. This is significant because it suggests that surface forces, no matter how small, may be important even for large particles.

\section{SIMULATION OF A SHEARED MONOLAYER}

In this section we apply the general method to the simulation of a monolayer of identical rigid non-Brownian spherical particles of radius $a$ in a simple shear flow. Figure 2 is a schematic diagram of the sheared monolayer showing that all particles lie in the plane of shear-the $(x, y)$ plane. The number of degrees of freedom for each sphere is reduced from six to three, resulting in an eightfold saving in computation time. Inverting the matrix $\mathbf{R}$ consumes the most time, growing as $N^{3}$; computing $\mathrm{R}$ grows only as $N^{2}$. While an 


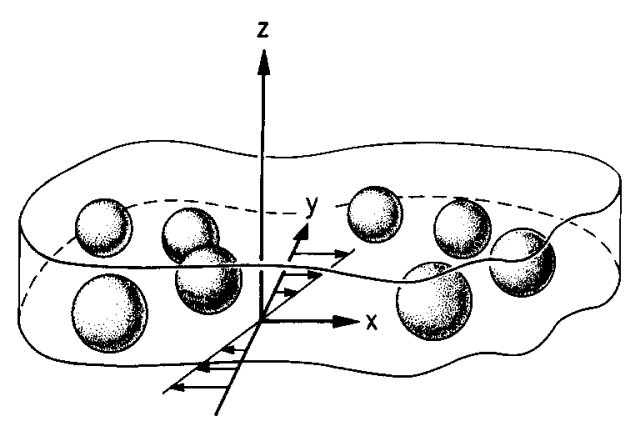

FIG. 2. Schematic diagram of a sheared monolayer. All spheres are of the same radius and lie in the plane of shear.

idealization, a monolayer is a dynamically possible suspension motion and experiments have been performed on such systems. ${ }^{15,16}$

In addition to the hydrodynamic interactions, the particles also interact through pairwise repulsive DLVO-type colloidal forces. There is a considerable body of literature on the nature of colloidal forces between particles in solution, and a valuable starting point is the classical work by Verwey and Overbeek. ${ }^{38} \mathrm{~A}$ simplified version of the presently accepted theory known as the DLVO theory holds that the colloidal forces are of two types: (1) a London-van der Waals attractive force; and (2) an electrostatic repulsive force due to the interaction between the particle double layers. In these simulations we have only included the repulsive double-layer forces because we wish in this first study to keep to a minimum the number of parameters to be varied. It is a simple (almost trivial) matter to include any type of interparticle force, and the effects of various forces on the local structure, on the suspension's rheology, and on problems of coagulation and flocculation can easily be investigated. It should be noted, however, that colloidal forces are most important for submicron size particles and, except at the highest shear rates, Brownian motion forces must also be considered.

For particles larger than a micron in size, the double layers are usually small compared with the particle radius, and following Takamura, Goldsmith, and Mason ${ }^{39}$ [their Eq. (6)] we can write the pairwise electrostatic repulsive force

$$
\mathbf{F}_{\text {rep }}=\mathbf{F}_{0} \frac{\tau e^{-\tau h}}{1-e^{-\tau h}},
$$

where $h=r-2$ is the separation between the sphere surfaces and $r$ is their center to center separation. Both have been made nondimensional with the sphere radius $a . \tau=\kappa a$ and $\kappa^{-1}$ is the Debye length. $F_{0}$ is the amplitude of the force and is given by

$$
\left|\mathbf{F}_{0}\right|=2 \pi \epsilon \psi^{2},
$$

where $\epsilon$ is the electrical permittivity of the fluid and $\psi$ is the surface potential of the particles when $h \rightarrow \infty$. The derivation leading to Eq. (6) assumes the surface potentials $\psi$ for the two particles are the same and the surface charge densities remain constant as the particles move relative to each other.

The important characteristics of this repulsive force are that it varies as $1 / h$ as $h \rightarrow 0$ and decays to $0\left(10^{-2}\left|\mathbf{F}_{0}\right| \tau\right)$ when $h \sim 0\left(4.5 \tau^{-1}\right) . \mathbf{F}_{0}$ sets the magnitude of the force and $\tau$ sets its range in space. At separations greater than $4.5 \tau^{-1}$ the inter- particle force will generally be negligible compared with the hydrodynamic force, and as $\tau \rightarrow \infty$ there is essentially no interparticle. Typical values of $\psi$ and $\tau$ taken from Takamura, et al..$^{39}$ for polystyrene latex spheres of $2 \mu \mathrm{m}$ radius in a $50 \%$ glycerol in water mixture containing $10^{-3} \mathrm{M} \mathrm{KCl}$ are: $\psi \sim 4 \times 10^{-3} \mathrm{~V}$ and $\kappa^{-1} \sim 88 \AA$, giving $\tau \sim 227$. The total interparticle force $\mathrm{F}_{p}$ is built up from Eq. (6) by considering all pairs of particles.

All lengths have been made nondimensional by the particle radius $a$. The elements of the grand resistance matrix are made nondimensional by $6 \pi \mu a$ when relating force to translational velocity, by $6 \pi \mu a^{2}$ when relating force to angular velocity, by $6 \pi \mu a^{2}$ when relating torque to translational velocity and by $6 \pi \mu a^{3}$ when relating torque to angular velocity. The elements of the shear resistance matrix are made nondimensional in the same way, except there is an additional power of $a$ in all elements because $\Phi$ multiplies $E$ rather than $U^{*}$. There are two choices for the velocity (and/or time) scale. The first comes from the shear rate and is $\gamma a$. The second is due to the interparticle forces $F_{\text {rep }}$ and is $\left|F_{0}\right| /$ $6 \pi \mu a$. We have chosen the latter scaling, and $U^{*}$ is made nondimensional with $2 \pi \epsilon \psi^{2} / 6 \pi \mu a$ and the time with $6 \pi \mu a^{2} /$ $2 \pi \epsilon \psi^{2}$. This scaling introduces the ratio of the shear to interparticle forces $6 \pi \mu a^{2} \dot{\gamma} / 2 \pi \epsilon \psi^{2}$, from which we define a dimensionless "shear rate"

$$
\dot{\gamma}^{*} \equiv \frac{6 \pi \mu a^{2} \dot{\gamma}}{2 \pi \epsilon \psi^{2}}
$$

Thus, in dimensionless form, Eq. (5) becomes

$$
\mathbf{U}^{*}=-\mathbf{R}^{-1} \cdot\left\{\boldsymbol{\gamma}^{*} \boldsymbol{\Phi}: \mathbf{E}+\mathbf{F}_{p}\right\},
$$

where all quantities are nondimensional. One can of course choose $\dot{\gamma} a$ as velocity scale and $\dot{\gamma}^{-1}$ as time scale, and this simply removes $\dot{\gamma}^{*}$ from in front of $\Phi$ and places $1 / \gamma^{*}$ in front of $\mathbf{F}_{p}$. This scaling is appropriate in the absence of any interparticle forces. From the experiments of Takamura, et $a l .{ }^{39}$ for the polystyrene latex spheres in the $50 \%$ glycerol in water mixture $6 \pi \mu a^{2} / 2 \pi \epsilon \psi^{2} \approx 6 s$; thus, $\dot{\gamma}^{*}=1$ corresponds to a shear rate of $1 / 6 s^{-1}$.

There are three dimensionless parameters which characterize the suspension: the areal fraction of particles $\phi$ defined as $\phi \equiv N \pi a^{2} / L H$, where $N$ is the number of particles in the periodic cell, $L$ is the length of the cell in the $x$ direction, and $H$ the length in the $y$ direction (cf. Fig. 2); the range of the interparticle force $\tau$; and, the ratio of the shear to interparticle forces $\gamma^{*}$. In addition to these physical parameters, there are two different pairwise additivity methods we wish to compare-superposition of forces and superposition of velocities. Since the superposition of forces entails inverting anew at each time step the resistance matrix, we shall refer to this method as "inversion." The superposition of velocities will be referred to simply as "superposition."

In all simulation runs the periodic cell was chosen to be square $(L=H)$. The particle trajectory equations were integrated in time in an explicit fashion. The simulations all started at $t=0$ with the particles located randomly in the periodic cell. The random initial positions were determined by first placing the spheres in a regular array and then displacing them a small random distance. The only constraint placed on the random displacements was that they not be so 
TABLE I. Presented are the relevant parameters for the 10 simulation runs that are discussed. Two simulation methods were employed: inversion-superposition of forces, and superposition-superposition of velocities. The other parameters are: the number of particles whose motion was followed, either 25 or 100 , the areal fraction $\phi$, the dimensionless ratio of shear to interparticle forces $\gamma$, the range of the interparticle forces $\tau$, the time step used $\Delta t$ and the total number of steps taken. $A$ " $2 \times$ " in front of the number of steps means that the run was repeated from different random initial positions.

\begin{tabular}{|c|c|c|c|c|c|c|c|}
\hline Run & Method & $\begin{array}{c}\text { Number } \\
\text { of } \\
\text { particles }\end{array}$ & $\phi$ & $\dot{\gamma}$ & $\tau$ & $\Delta t$ & $\begin{array}{c}\text { Number } \\
\text { of } \\
\text { steps }\end{array}$ \\
\hline$A$ & inversion & 25 & 0.4 & 0.5 & 227 & $4 \times 10^{-3}$ & $2 \times 20000$ \\
\hline$B$ & superposition & 25 & 0.4 & 0.5 & 227 & $2 \times 10^{-3}$ & $2 \times 40000$ \\
\hline$C$ & superposition & 100 & 0.4 & 0.5 & 227 & $2 \times 10^{-3}$ & $2 \times 40000$ \\
\hline$D$ & inversion & 25 & 0.4 & 0.5 & no force & $4 \times 10^{-3}$ & $2 \times 20000$ \\
\hline$E$ & inversion & 25 & 0.4 & 0.5 & 10 & $4 \times 10^{-3}$ & 20000 \\
\hline$F$ & inversion & 25 & 0.57 & 0.5 & 10 & $2 \times 10^{-3}$ & 2500 \\
\hline$G$ & inversion & 25 & 0.57 & 0.5 & 50 & $4 \times 10^{-3}$ & 16000 \\
\hline $\boldsymbol{H}$ & inversion & 25 & 0.1 & 1 & 227 & $4 \times 10^{-3}$ & $2 \times 20000$ \\
\hline$I$ & superposition & 25 & 0.1 & 1 & 227 & $2 \times 10^{-3}$ & 80000 \\
\hline$J$ & superposition & 100 & 0.1 & 1 & 227 & $2 \times 10^{-3}$ & $2 \times 40000$ \\
\hline
\end{tabular}

large as to cause particles to overlap initially. The particle motions were followed in time until a stationary state was reached. The stationariness was determined by monitoring the average of the square of the $x$ and $y$ components of the particle velocities relative to the bulk fluid, i.e., $\left\langle U_{y}^{2}\right\rangle$ and $\left\langle\left(U_{x}-\dot{\gamma}^{*} y\right)^{2}\right\rangle$, where the averages are over both particles and time. Generally, 20000 time steps yielding a dimensionless time of $0(80)$ were used, and most simulations were run twice from different random initial conditions in order to estimate the magnitude of the statistical errors. Most runs were carried out with 25 particles, as this number yields affordable computation time and still contains enough particles to include all second nearest neighbors. (Assuming hexagonal close packing in a plane, a central particle has six nearest neighbors and 12 next nearest neighbors.) For an areal fraction $\phi=0.4$ and 25 particles the edge of the periodic cell is 3.5 particle diameters from a central particle which, as we shall see, is greater than the range of $g(\mathbf{r})$. All computations were carried out in double precision on an IBM 3033.

A summary of the 10 runs to be discussed in Sec. IV is shown in Table I, giving the method used-superposition or inversion, the number of particles, the physical parameters $\phi, \tau$, and $\gamma$ (the ${ }^{*}$ on $\dot{\gamma}$ has been dropped for convenience), the time step, and the number of steps taken. A " $2 \times$ " in front of the number of steps means the run was repeated from a different random initial condition. Computation times for inversion are significantly greater than those for superposition. The total computation time required to reach a dimensionless time of 80 in run $A$ was $135 \mathrm{~min}$, while in $\operatorname{run} B$ it was $4.5 \mathrm{~min}$. In the superposition method it was necessary to use a smaller time step and a predictor-corrector method to insure nonoverlapping of particles. Hence, for the same total dimensionless time there is at least a factor of 30 between the two methods.

\section{RESULTS AND DISCUSSION}

The simulation results will be presented principally in terms of the pair distribution function $g(\mathbf{r}) \cdot g(\mathbf{r})$ is defined as the probability of finding a particle center at position $r$ given that there is a particle center at the origin $\mathbf{r}=0$ divided by the number density. Thus, $g(\mathbf{r}) \sim 1$ as $|\mathbf{r}| \rightarrow \infty$. In the statistical mechanics literature $g(\mathbf{r})$ is often called the pair correlation function and $g$ of scalar $r$ is known as the radial distribution function. In sheared suspensions $g(\mathbf{r})$ has in general both an angular and a radial structure, i.e., $g(\mathbf{r})=g(r, \theta)$, where $r=|\mathbf{r}|$ and $\theta$ is the angle measured from the $x$ axis. $\theta=0$ corresponds to the downstream side of the reference particle and $\theta=180^{\circ}$ to the upstream side. The inset in Fig. 3 should help clarify the meaning of $\theta$. The symmetry of the simple shear flow is such that

$$
g(r, \theta)=g(r, \theta+\pi) \text {. }
$$

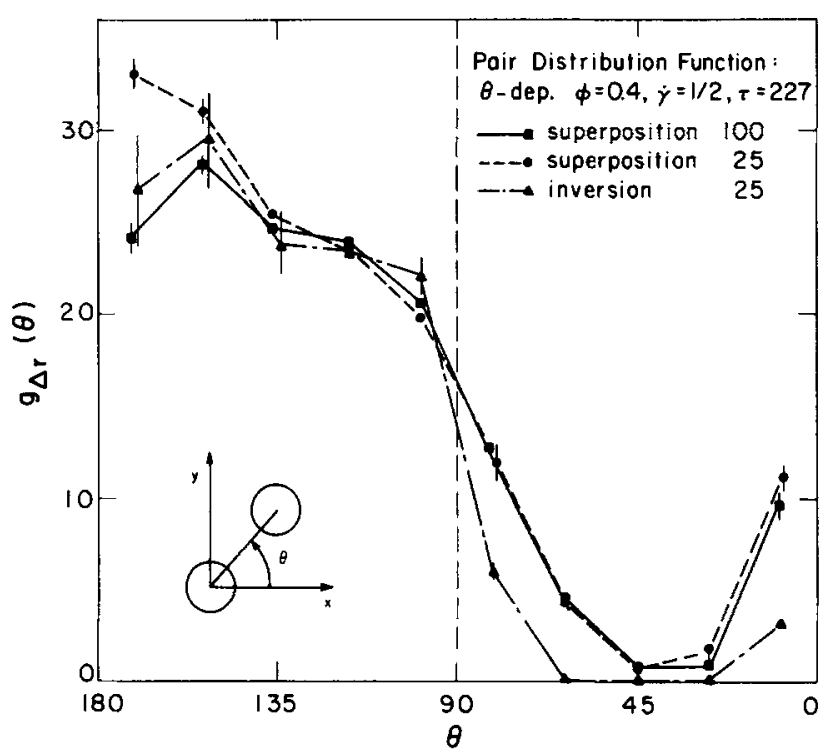

FIG. 3. The angular dependence of the pair distribution function. $g_{\Delta_{r}}(\theta)$ is the probability of finding a particle center in the range $2<r<2.03$ given that there is a particle center at the origin. All three curves are at the same conditions of $\phi=0.4, \dot{\gamma}=1 / 2$, and $\tau=227$, but correspond to different simulation methods or to a different number of particles. From Table I, the runs are as follows: $\longrightarrow \triangle A, \cdots B$, and $\rightarrow C$. The inset defines the angle $\theta$ relative to the $x$ axis. $0^{\circ}<\theta<90^{\circ}$ corresponds to the downstream side of the reference sphere and $90^{\circ}<\theta<180^{\circ}$ to the upstream side. The angular structure is a result of the repulsive interparticle forces. 
To determine the pair distribution function the region about the reference particle was divided into ten angular wedges each spanning $18^{\circ}$, with the first wedge centered at $9^{\circ}$ and the last at $171^{\circ}$. The radial dependence has an excluded volume region for $r<2$, followed by a very pronounced peak close to the minimum separation distance of $r=2$ and a much weaker peak corresponding to the second nearest neighbors. To resolve these two disparate peaks, $g(r, \theta)$ was determined twice with two different $\Delta r$ increments, 0.01 and 0.05 . The value of $g(r, \theta)$ in a given region of size $\Delta r \Delta \theta$ was determined by averaging over time the number of particle centers found in this region. The time averaging was done after the system had reached a stationary state. Because of both the $\theta$ and $r$ dependence, presenting $g(r, \theta)$ in a concise format is difficult. We have chosen to present an overall view of the angular structure near the minimum separation distance and radial distributions at four values of $\theta, \theta=9^{\circ}, 27^{\circ}$, $99^{\circ}$, and $171^{\circ}$.

Figure 3 shows the angular distribution $g_{\Delta r}(\theta)$ for runs A, B, and C in Table I. $g_{\Delta r}(\theta)$ is the probability of finding a
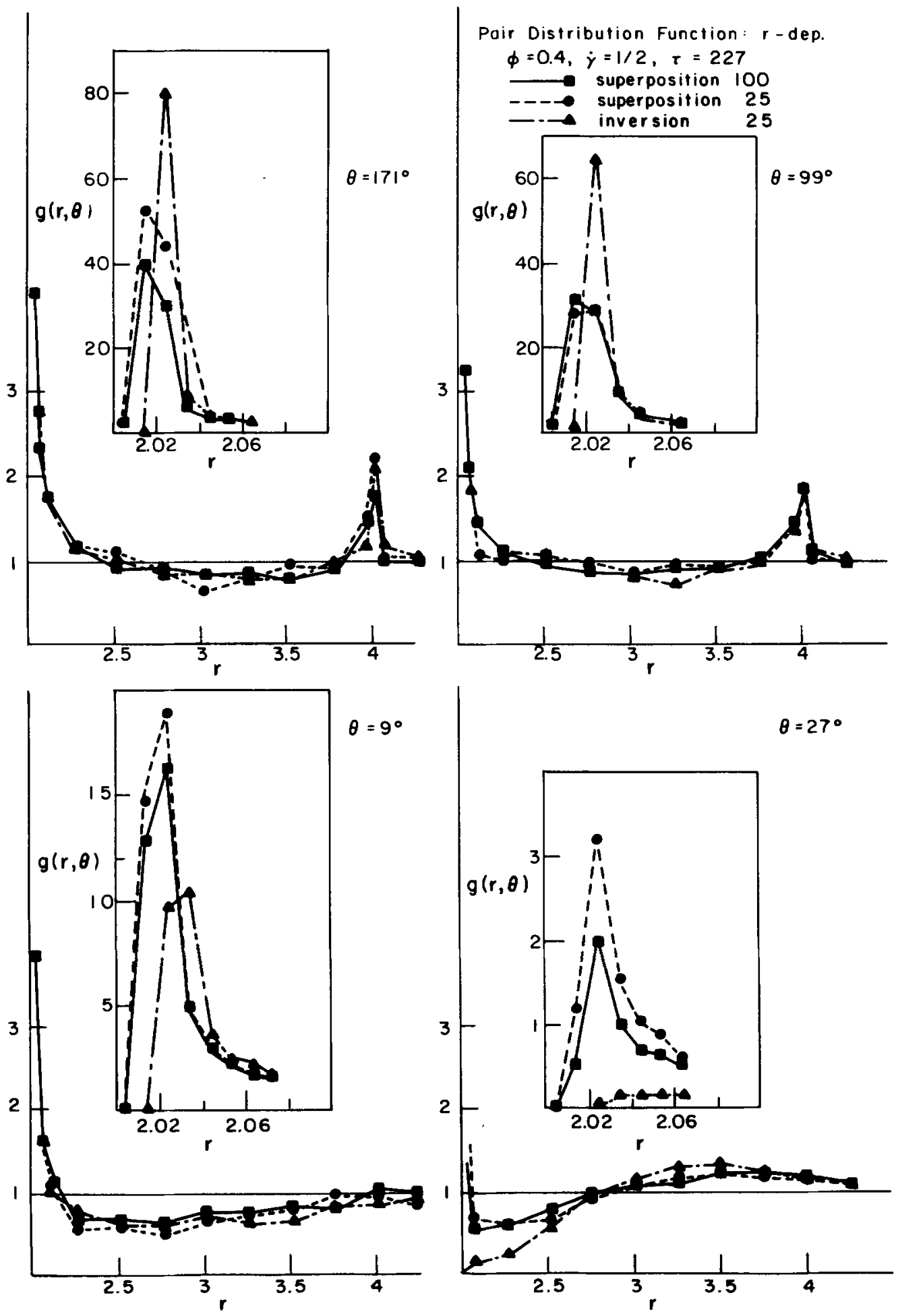

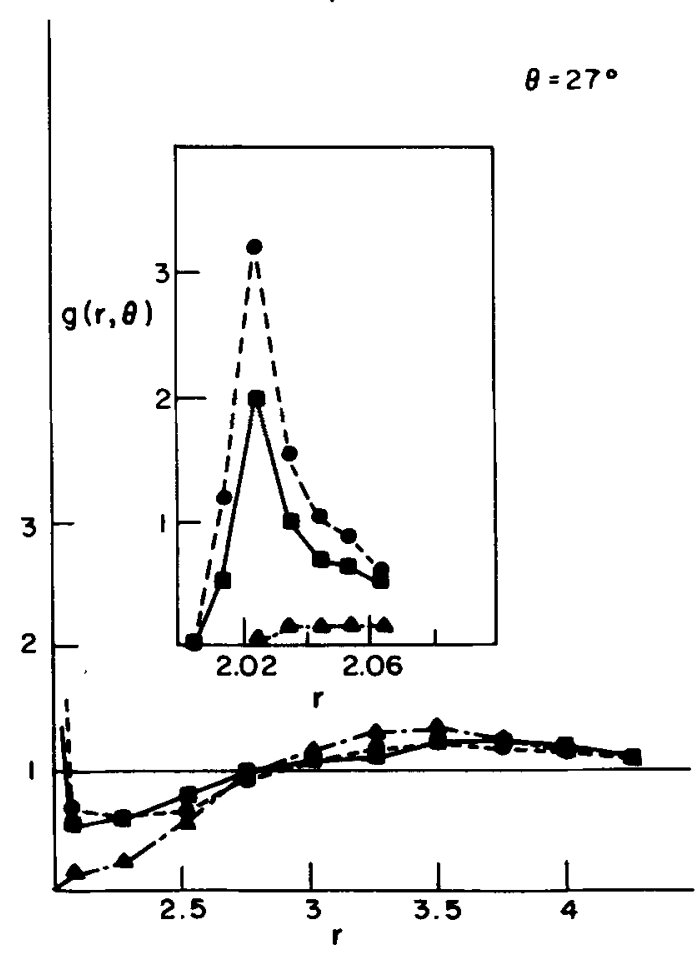

FIG. 4. The radial dependence of the pair distribution function for the same runs as in Fig. 3 at four values of $\theta: \theta=9^{\circ}, 27^{\circ}, 99^{\circ}$, and $171^{\circ}$. The inserts show the detail of the first nearest neighbor peak, while the larger scale shows the peak due to the second nearest neighbors. 


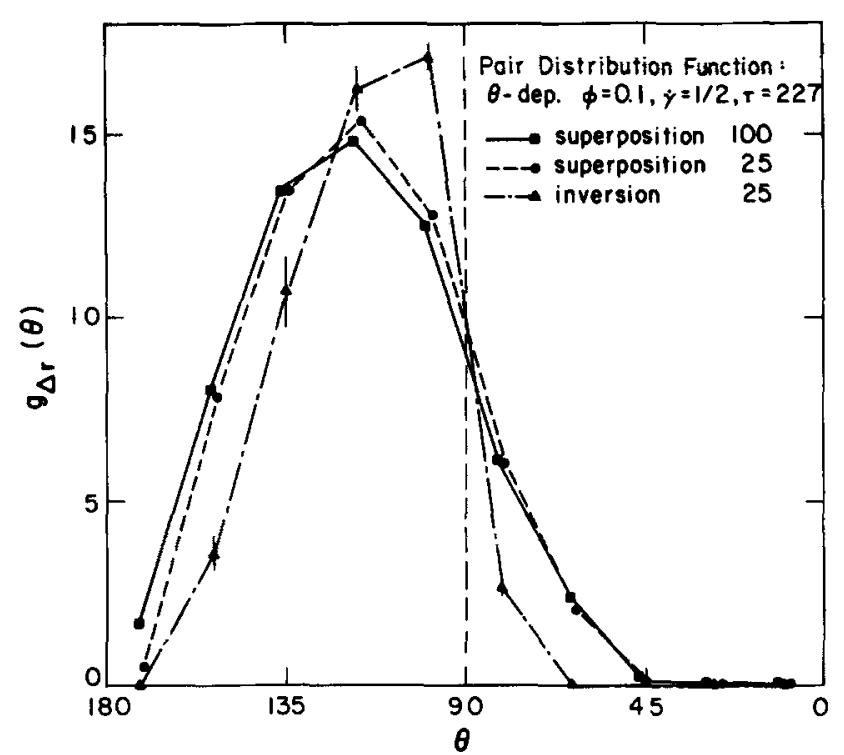

FIG. 5. The angular dependence of the pair distribution function as in Fig. 3 but at a lower areal fraction $\phi=0.1$. From Table I the runs are as follows: $\longrightarrow \mathrm{H}, \rightarrow \mathrm{I}$, and $\longrightarrow \mathrm{J}$. second particle in the region $2<r<2.03$. A $\Delta r$ of 0.03 was chosen because this distance extends out past the range of the interparticle force $\left(4.5 \tau^{-1} \approx 0.02\right)$ and encompasses the first nearest neighbor peak. An areal fraction of $\phi=0.4$ is a reasonably concentrated suspension. The maximum flowing fraction is $\phi_{\max }=\pi / 4 \approx 0.785$. At $\dot{\gamma}=1 / 2$ the shear and interparticle forces are of the same magnitude. The error bars shown in Fig. 3 were determined by comparing the two separate runs at different random initial positions. The solid points are the average values and if no error bars are shown they are no larger than the symbol size.

Figure 3 shows that there is quite good agreement between superposition with 25 and 100 particles, indicating that 25 particles give accurate distribution functions. While not quantitative the agreement between inversion and superposition with 25 particles is qualitatively good. In all cases there is a pronounced angular structure with a very few particles on the downstream side, $0^{\circ}<\theta<90^{\circ}$, of the reference sphere and a high concentration on the upstream side $90^{\circ}<\theta<180^{\circ}$. The explanation of this angular structure is

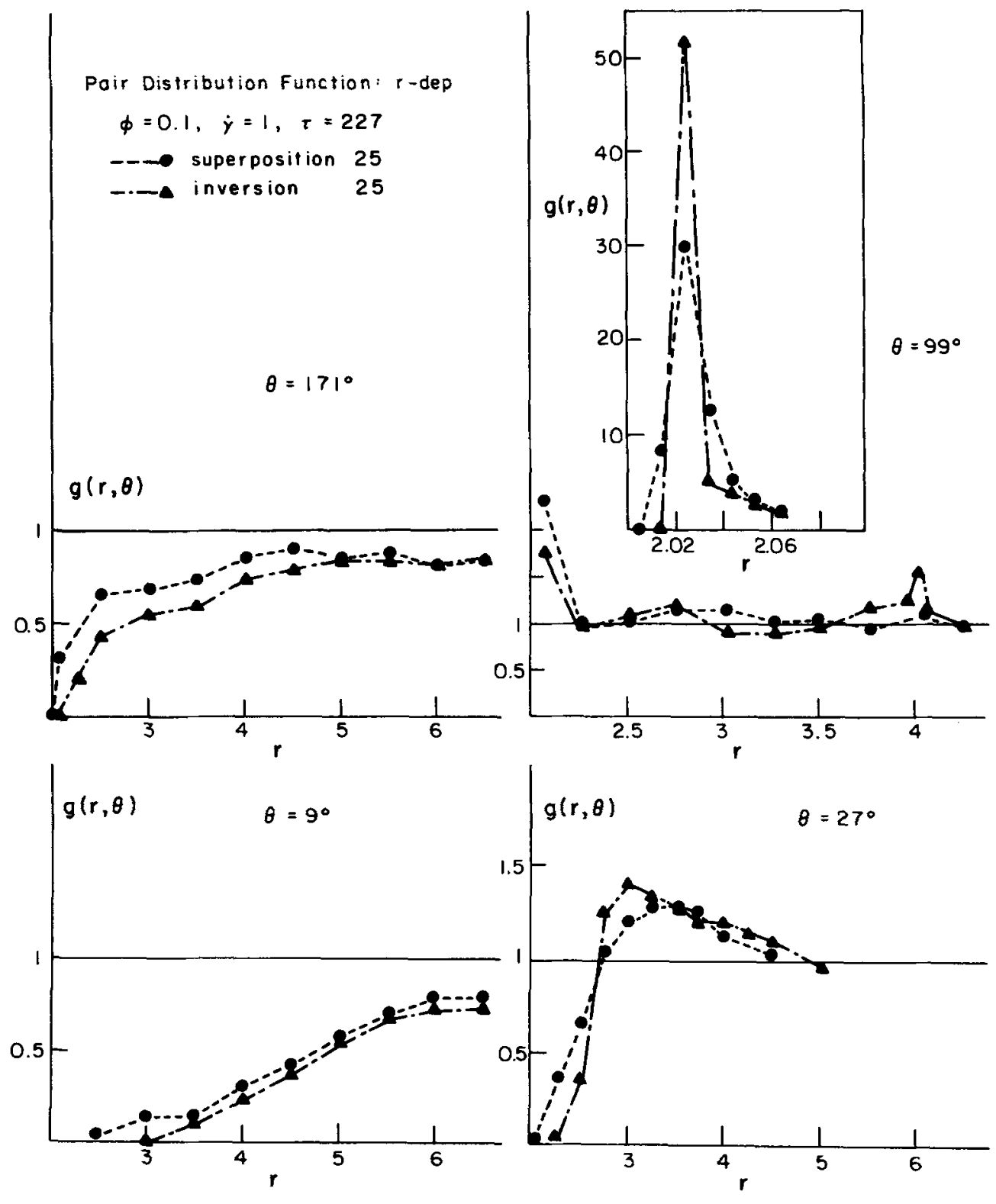

FIG. 6. The radial dependence of the pair distribution function for the runs $H$ and $I$ of Fig. 5. 


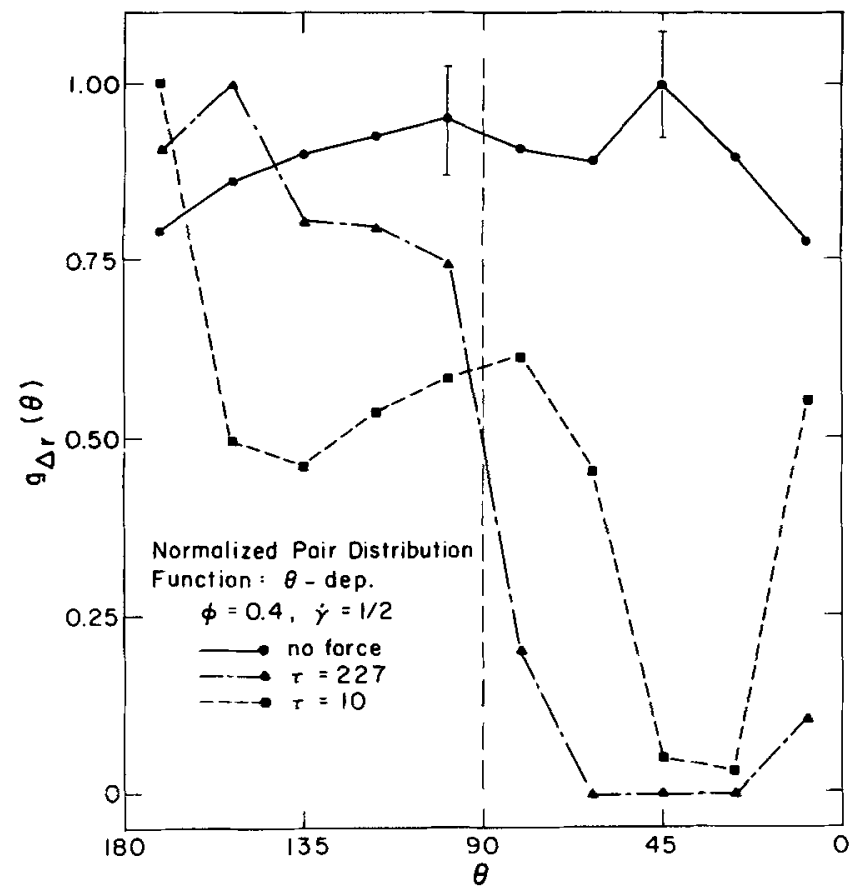

FIG. 7. A comparison of the angular dependence of the pair distribution function for different ranges of interparticle force. Each $g_{\Delta r}(\theta)$ has been normalized by its maximum value and the $\Delta r$ ranges vary with $\tau: \tau=10$, $2<r<2.3 ; \tau=227,2<r<2.03$; and in the absence force, $2<r<2.01$. Table $I$ runs are: $--E$, $\longrightarrow \Delta A$, and $\rightarrow D$. Without interparticle forces there is no angular structure.

quite simple. On the upstream side the shear forces are pushing the two particles together while the repulsive force is typing to keep them apart. On the downstream side both the shear and repulsive forces are acting to separate the particles. A similar "depletion" region is observed in two-sphere trajectories. ${ }^{39}$ The asymmetry in the angular structure is due to the repulsive interparticle force, as the pair hydrodynamic forces have symmetry about $\theta=90^{\circ}$. The apparent lack of agreement of $g_{\Delta r}$ at $0^{\circ}$ and $180^{\circ}$ is a statistical error due to the way in which the interval $0^{\circ} \leqslant \theta \leqslant 180^{\circ}$ has been subdivided and to the depletion region on the downstream side.

Figure 4 shows the radial dependence of the distribution function for the same runs A, B, and $C$ as in Fig. 3 . Starting in the lower left-hand corner and proceeding in a counter clockwise direction moves around in $\theta$ from the downstream to the upstream side. Two different scales are shown in order to resolve both the first and second nearest neighbor peaks. Again, a comparison is made between superposition with 25 and 100 particles, the agreement generally being quite good. Note, this is a more stringent test than in Fig. 3 as the comparison is on a pointwise basis. Agreement between inversion and superposition is again qualitative, but the quantitative differences become more evident. In particular, the intensity of the first nearest neighbor peaks, their width and location are all slightly different. In general the inversion peaks occur at larger $r ; 2.025$ as compared with 2.02 . This is due to the fact that the lubrication forces are properly represented by inversion but not by superposition. This may seem a small matter, but the slight variations in the location and width of the peak may have a large effect on the rheological properties of this suspension.
We also see that the first nearest neighbor peaks are much weaker on the downstream side. The minimum intensity usually occurs near $\theta=45^{\circ}$ where the shear forces change from pulling particles apart $90^{\circ}<\theta<45^{\circ}$ to bringing them together $45^{\circ}<\theta<0^{\circ}$; the hydrodynamic forces vary as $\sin 2 \theta$. The second nearest neighbor peaks are all very weak and occur at the second excluded volume distance $r=4$. Note the rapid approach of the pair distribution function to unity.

Superposition of velocities and superposition of forces (inversion) give qualitatively the same behavior at $\phi=0.4$. To see if this agreement would improve at lower densities where the lubrication forces would not be as important, simulations were performed at $\phi=0.1$, runs $H, I$, and $J$ in Table I. Figure 5 shows the angular dependence of the distribution function for the same $\Delta r$ of 0.03 as in Fig. 3. There is again a large angular structure, although not as pronounced as at $\phi=0.4$. Figure 6 shows the radial dependence for runs $H$ and $I$. The structure here is radically different from that at $\phi=0.4$, there being a first nearest neighbor peak only near $\theta=90^{\circ}$. Clearly, density has a profound effect on the local structure. It is also apparent that the agreement between superposition and inversion has not improved markedly at the lower density. This is important from a computation time point of view, and we have not as yet determined the precise conditions under which one can use with confidence and accuracy the superposition of velocities. These conditions may very well depend upon what property is being computed.

Figures 7 and 8 show the importance of the range of the interparticle force. These results correspond to runs $A$, $D$, and $E$. The $g_{\Delta r}(\theta)$ in Fig. 7 are all normalized by their respective maximum values for comparison. The ranges $\Delta r$ are different, as each is chosen to encompass the first peak: $\tau=10, \Delta r=0.3 ; \tau=227, \Delta r=0.03$; and with no force $\Delta r=0.01$, the minimum increment used in computing $g(r, \theta)$. As mentioned in Sec. III, when there is no interparticle force particles overlap slightly from time to time. Thus, the probability of finding a particle center in the range $1.99<r<2.00$ is nonzero, and these overlapped particles are considered to be at $r=2$.

Figure 7 shows there is a dramatic change in angular structure with interparticle force. In the absence of any such force there is essentially no angular structure, reflecting the symmetry of the hydrodynamic forces. The error bars are the same order as the variation observed from $0^{\circ}$ to $180^{\circ}$. The $\tau=227$ results are the same as in Fig. 3 and show clearly that the angular structure is caused by the repulsive forces. As the range of the interparticle force increases $(\tau=10)$, the angular distribution appears to develop more structure. This evolution in structure is related to the transition to an ordered layered structure that occurs at high $\phi$ and/or low $\tau$ (see below).

The radial dependence is shown in Fig. 8. Without interparticle forces the first peak occurs right at $r=2$ and is quite large, on the order of 100 . As the interparticle forces increase in range the nearest neighbor peak is pushed away from the excluded volume surface $r=2$. Somewhat surprisingly, the first peak at $\tau=10$ is more pronounced than that 

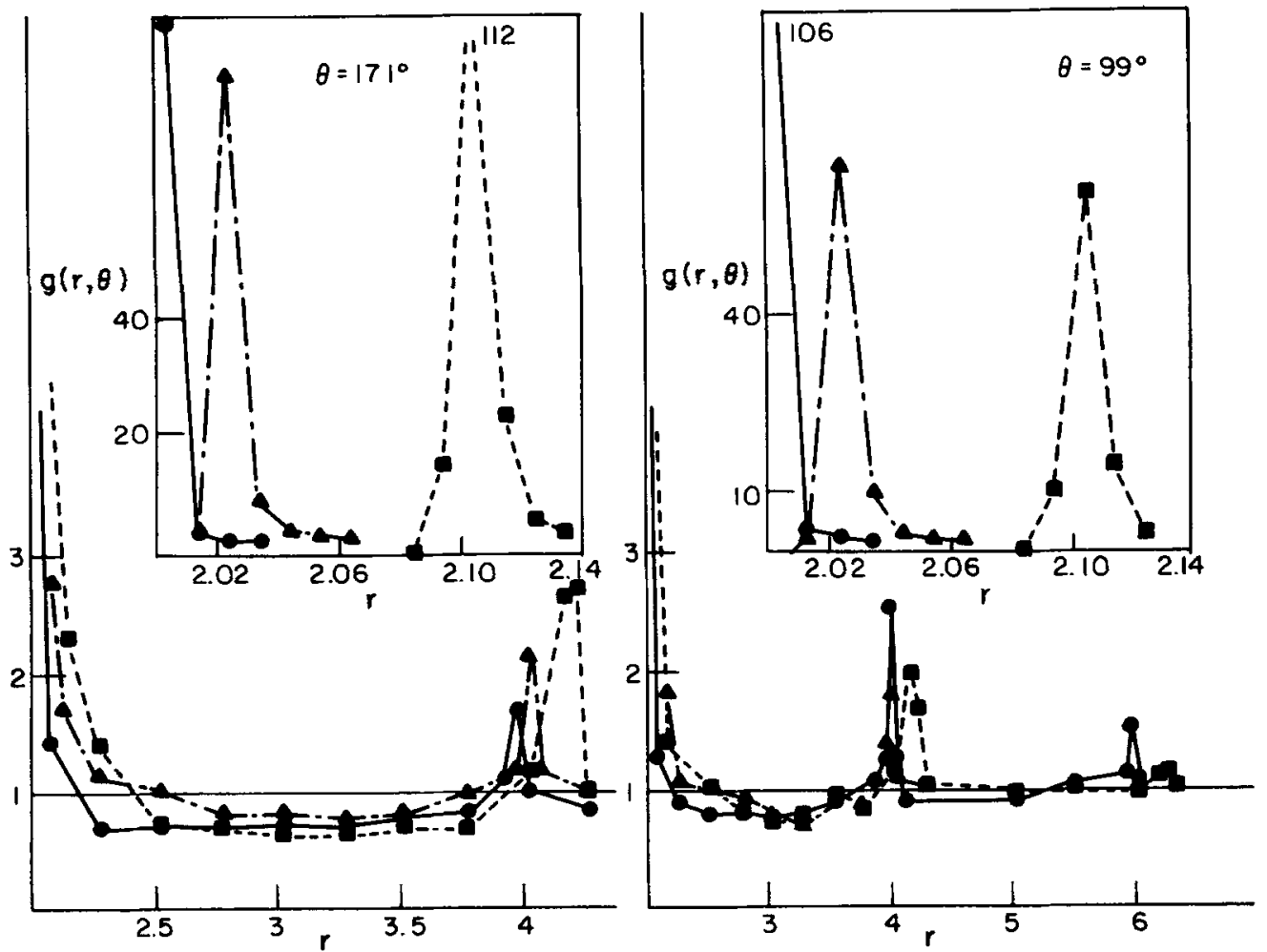

FIG. 8. A comparison of the radial dependence of the pair distribution functions for the same runs as in Fig. 7.
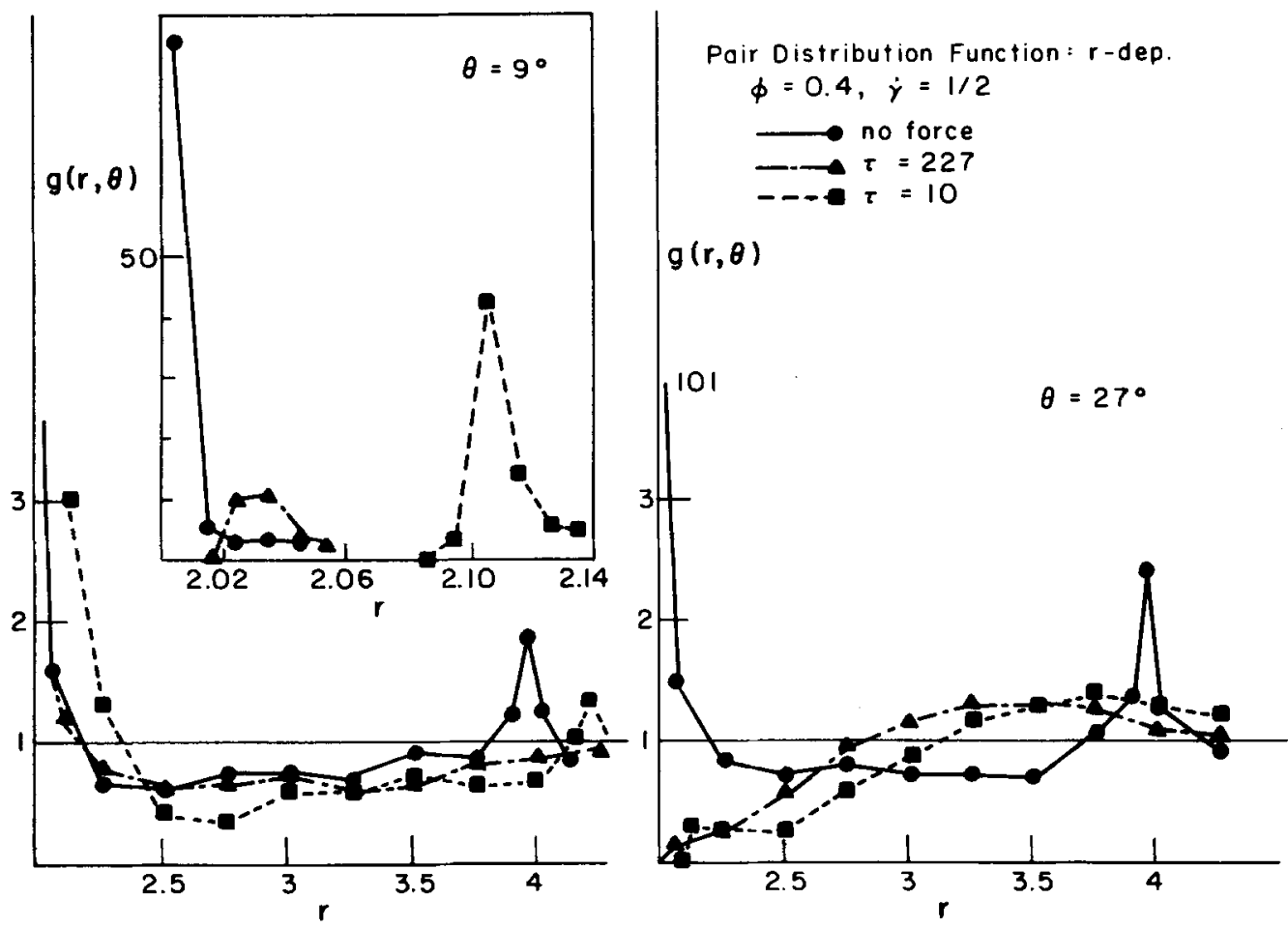

at $\tau=227$, whereas in a classical liquid a less abrupt (smaller $\tau)$ force gives rise to a weaker peak. The second nearest neighbor peaks are relatively unaffected. At $\theta=99^{\circ}$ we can also see the presence of the third nearest neighbor peak at $r \approx 6$. Figure 8 shows the absence of an angular structure without interparticle forces, and the large effect the repulsive forces have on the downstream side.

In a sheared suspension the maximum volume fraction that can flow is always less than the maximum packing fraction. Near the maximum flowing fraction the suspension is in a highly ordered state, as planes of particles slide over one another. This layered structure can be approached either by increasing the number density of particles or by increasing the range of the interparticle force or by both. The interparticle forces create a larger "effective" particle radius which leads to a larger effective volume fraction. Figure 9 shows what happens on increasing the concentration and range of interparticle force; the results are from runs $A, G$, and $F . g(y)$ is defined as the probability of finding a particle center in the plane $y=$ const. parallel to the $x$ axis given that there is a 

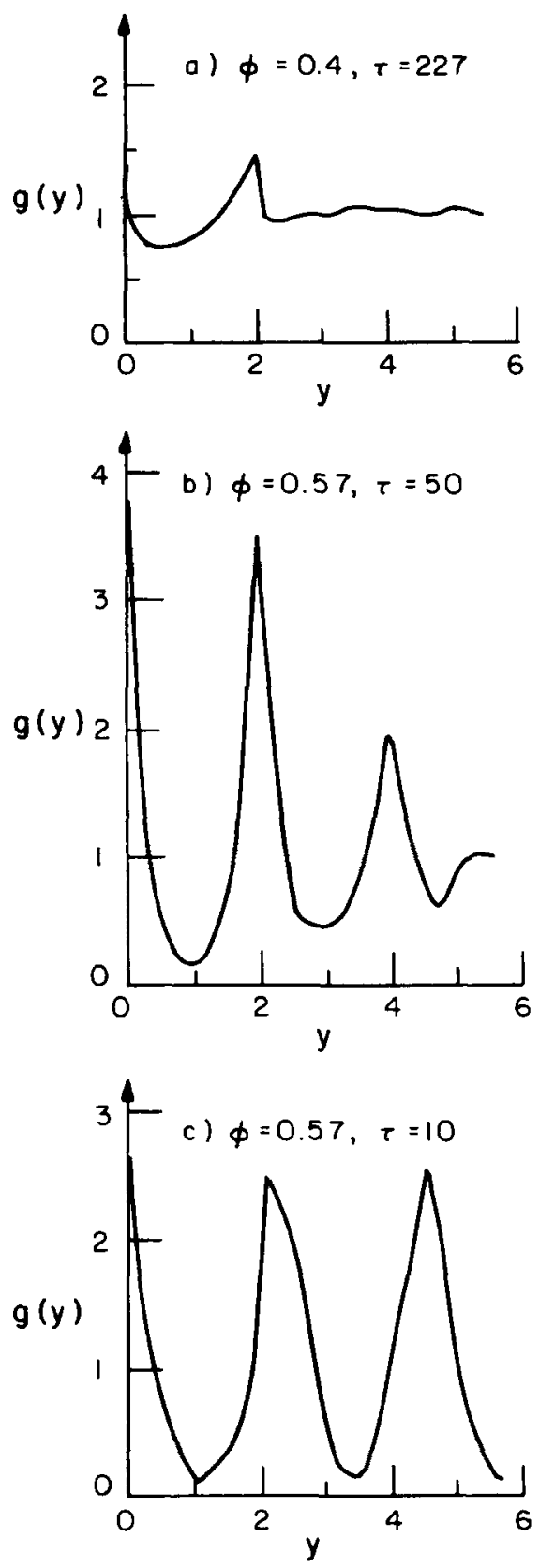

FIG. 9. Results showing a transition to an ordered layered structure upon increasing areal fraction and range of interparticle force (decreasing $\tau$ ). The maximum areal fraction able to flow is $\phi_{\max }=\pi / 4 . g(y)$ is the probability of finding a particle center on the line $y=$ const. parallel to the $x$ axis. All results are at $\gamma=1 / 2$. (a) $\phi=0.4, \tau=227$, run $A$ showing no layered structure. (b) $\phi=0.57, \tau=50$, run $G$. A layered structure is forming. (c) $\phi=0.57, \tau=10$, run $F$. The layered structure is completely formed. The repulsive interparticle force results in an effective particle size and areal fraction near the maximum allowable even though the actual $\phi$ is only 0.57 .

particle center at the origin. In Fig. 9(a) we see clearly the first nearest neighbor peak at $y=2$, but there is no structure beyond that. Increasing the areal fraction to $\phi=0.57$ and the range of the interparticle force [Fig. 9(b)], we see the development of a layered structure with particles regularly spaced in the $y$ direction. By increasing the range of the interparticle force still further [Fig. 9(c)], the transition to a layered structure is complete. Figure 10 shows the instantaneous configuration of the suspension in Fig. 9(c) at a dimen-

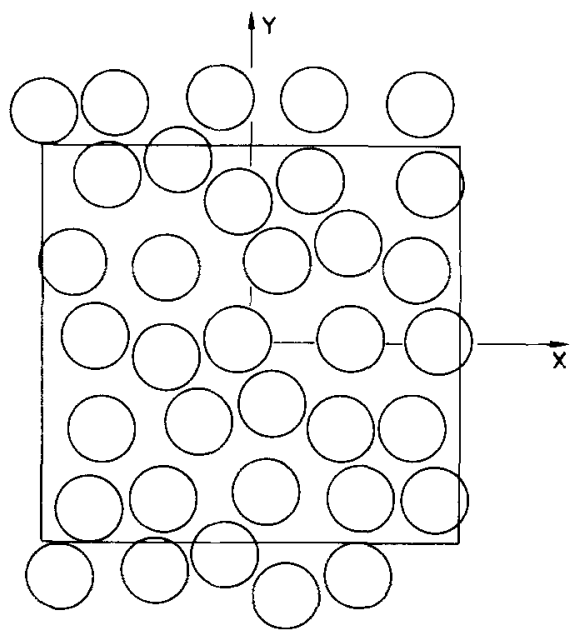

FIG. 10. Instantaneous particle configuration corresponding to the conditions of Fig. 9(c). The particles outside the periodic cell are some of the closest images. Careful observation will reveal the layered arrangement in the $y$ direction.

sionless time of approximtely five. Viewed carefully one can see the layers of particles in the $y$ direction.

The "effective" particle radius due to the repulsive forces can be estimated by finding the separation $h$ at which the shear and interparticle forces balance, i.e.,

$$
\frac{\tau e^{-\tau h}}{1-e^{-\tau h}}=\dot{\gamma} \Phi_{\mathrm{rad}}(h) \text {. }
$$

Here, $\Phi_{\text {rad }}(h)$ is the component of the shear resistance matrix acting along the line of centers between two particles averaged over the upstream side $90^{\circ}<\theta<180^{\circ} .^{40}$ With $\dot{\gamma}=1 / 2$, $\tau=10$, estimating $h$ from Eq. (10) gives $h \approx 0.36$. Hence, the particles have an effective radius of 1.18 , and hence an effective areal fraction

$$
\phi_{\text {eff }}=(0.57)(1.18)^{2}=0.794 \text {. }
$$

This is to be compared with the maximum flowing areal fraction

$$
\phi_{\max }=\pi / 4 \approx 0.785 \text {. }
$$

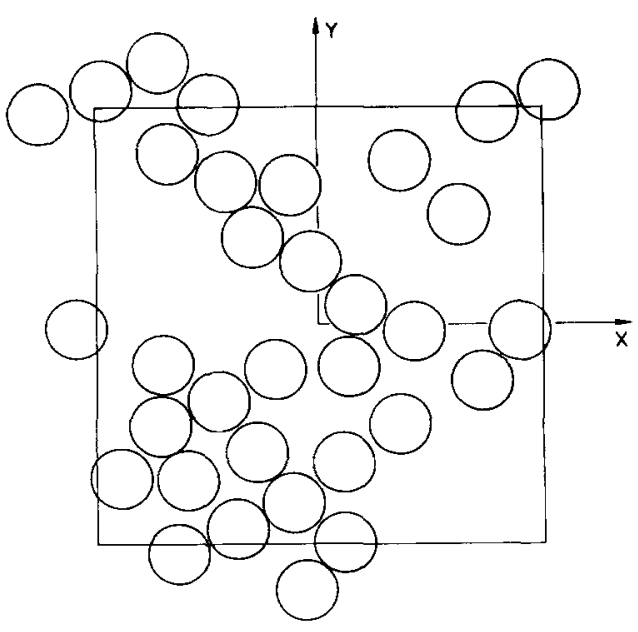

FIG. 11. Typical particle configuration for $\phi=0.4, \dot{\gamma}=1 / 2$ in the absence of interparticle forces (run $D$ ). Note the region devoid of particles and the presence of a cell spanning cluster. 


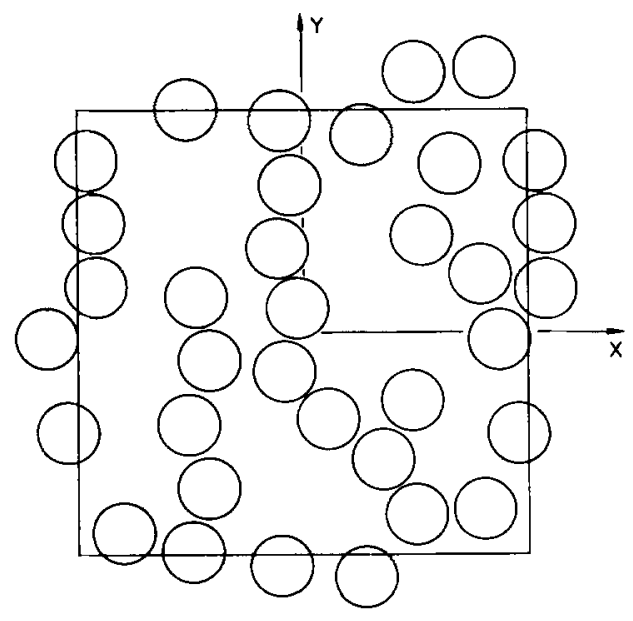

FIG. 12. Same as Fig. 11 but with interparticle forces, $\tau=227$ (run $A$ ). Note the lack of any void regions and the much smaller cluster size.

While highly simplified, the effective radius given by Eq. (10) seems to contain the essential physics. The change from a purely local to a layered structure on increasing $\Phi_{\text {eff }}$ is reminiscent of a phase transition, but it is not known whether there exists a well-defined critical areal fraction. This transition can also have a profound effect on the suspension "viscosity." Experiments of Hoffman ${ }^{41}$ have shown a discontinuous viscosity behavior with shear rate (changing the relative magntiude of the shear and interparticle forces) and volume fraction which he interpreted as due to a layereddisordered transition.

As a final illustration of the behavior of concentrated suspensions, in Figs. 11 and 12 we show typical instantaneous configurations at arbitrarily chosen times for two different runs. Figure 11 corresponds to run $D$ in which there are no interparticle forces, and Fig. 12 is at the same $\phi$ and $\dot{\gamma}$, but with interparticle forces, run $A$. One is struck by the large void regions in Fig. 11 and by what appears to be a cell spanning cluster. In Fig. 12 there are also clusters of particles but their characteristic size is consideraby smaller and none span the periodic cell. These clusters are dynamic with particles continually breaking off, forming new clusters, and rejoining the original cluster in a different region. It should be noted that the large cluster in Fig. 11 evolves but persists in time; it is not an artifact of the specific instant in time we have chosen to represent. These large clusters are intriguing because we are seeing structure on a much larger scale than the local structure we have been discussing up to this point. The dynamics of these large structures and their influence on the macroscopic behavior of suspensions are not known. Analogies with percolation ideas come to mind, and it has been conjectured that an infinite cluster may occur at a critical volume fraction. ${ }^{42}$ Unfortunately, periodic boundary conditions are not proper for investigating "infinite" clusters, because a cell spanning one is necessarily infinite. Furthermore, the imposed bulk linear shear flow necessitates that all particles move in accordance with the bulk velocity gradient and thus no "plug" flow region may form. To investigate the possible transition with volume fraction to an infinite cluster and the resulting suspension rheology, a simulation with boundaries must be performed.

\section{CONCLUSIONS}

In this paper we have presented a rather general method for simulating the dynamical behavior of a suspension of hydrodynamically interacting particles. The method uses periodic boundary conditions to represent an infinite suspension and two methods were used for treating the hydrodynamic interactions among particles. Both the pairwise superposition of forces and pairwise superposition of velocities are in qualitative agreement as regards the shear induced anistropic local structure. The superposition of forces is the preferred method because it models properly the lubrication forces which prevent particles from overlapping. The superposition of velocities cannot be used at all if there are no strong repulsive interparticle forces.

In the numerical simulation of a monolayer of spheres, it was seen that the shear induced local structure has, in general, both an angular and a radial dependence. The angular dependence was shown to be caused by the repulsive interparticle forces, while the radial structure is primarily an excluded volume effect. Both the angular and radial structure depend strongly on the concentration of particles, the range of the interparticle forces and the shear rate. It was also shown that by increasing the concentration of particles up to near the maximum areal fraction that can flow and/or by increasing the range of the interparticle force results in a transition to a layered structure.

The details of the local structure depend on a delicate balance of shear and interparticle forces. It was shown that the transition to the layered structure with increasing range of interparticle force could be explained in terms of an "effective" particle radius given by the point where the average shear and interparticle forces just balance. This notion of an effective radius has a good physical basis and can be applied to estimate the location of the first nearest neighbor peaks shown in Fig. 8. For $\tau=227$, Eq. (10) gives a separation $r=2.017$, while the first peak is at $r=2.025$. The agreement is reasonable but not complete. For $\tau=10$, however, Eq. (10) gives a separation of $r=2.36$, while the first peak is at $r=2.1$. Here the agreement is quite poor and really indicates that without the layered structure (none was observed for run $E$ ), particles are on average closer together than their effective radius would predict. They are actually closer together at $\phi=0.4$ than at $\phi=0.57$. This last example shows that the location of the first peak involves a more subtle interplay of shear and interparticle forces than represented by Eq. (10).

In this paper we have not discussed the rheological properties of suspensions which can also be determined by this simulation method. Our purpose was to present a method and to illustrate the types of structural behavior to be found in sheared suspensions. Our preliminary calculations on the "effective" viscosity show general agreement with experiment, but the detailed results must await a future study in which we address this question. Work is also in progress on the transition to a layered structure and the dynamics of the cell spanning cluster. It should be evident that the generality of the method allows one to investigate many different suspension problems. And its extension to include 
Brownian motion and boundary effects opens up even further possibilities.

\section{ACKNOWLEDGMENTS}

We would like to express our appreciation to E. Guyon who helped initiate this work by bringing us together and who has since followed our effort with considerable interest. This research was supported in part by a grant from the Centre National de la Recherche Scientifique under the A.T.P. program in macroscopic random materials and by a grant from Monsanto Company. This work was initiated when J.F.B. was in France under the auspices of the NSF/ CNRS Exchange of Scientists Program, and this support is gratefully acknowledged. This paper is dedicated to our friend and colleague Bernard Quentrec who began this project with us, but met an untimely death before seeing it completion.

'G. K. Batchelor, Annu. Rev. Fluid Mech. 6, 227 (1974).

${ }^{2}$ H. Brenner, Int. J. Multiphase Flow 1, 195 (1974).

${ }^{3}$ D. J. Jeffrey and A. Acrivos, AIChEJ 22, 417 (1976).

${ }^{4}$ W. B. Russel, J. Rheol. 24, 287 (1980).

${ }^{5}$ P. Mazur and W. van Saarloos, Physica A 115, 21 (1982),

${ }^{6} \mathrm{~W}$. van Saarloos and P. Mazur, Physica A 120, 77 (1983)

${ }^{7}$ C. W. J. Beenakker and P. Mazur, Physica A 120, 338 (1983).

${ }^{8}$ M. Zuzovsky, P. M. Adler, and H. Brenner, Phys. Fluids 26, 1714 (1983).

${ }^{9}$ P. M. Adler and H. Brenner, Phys. Fluids (submitted).

${ }^{10}$ P. M. Adler, M. Zuzovsky, and H. Brenner, Phys. Fluids (submitted).

"A. A. Zick and G. M. Homsy, J. Fluid Mech. 115, 13 (1982).

${ }^{12}$ A. S. Sangani and A. Acrivos, Int. J. Multiphase Flow 8, 343 (1982).

${ }^{13}$ D. L. Ermack and J. A. McCammon, J. Chem. Phys. 69, 1352 (1978).

${ }^{14} \mathrm{~J}$. Bacon, E. Dickinson, and R. Parker, J. Chem. Soc. Faraday Trans. 279, 91 (1983).

${ }^{15}$ M. Belzons, R. Blanc, J. L. Bouillot, and C. Carmoin, C. R. Acad. Sci.
Paris 292, 939 (1981).

${ }^{16}$ J. L. Bouillot, C. Camoin, M. Belzons, R. Blanc, and E. Guyon, Adv. Colloid Interface Sci. 17, 299 (1982).

${ }^{17} \mathrm{~J}$. Happel and H. Brenner, Low Reynolds Number Hydrodynamics (Prentice-Hall, Englewood Cliffs, New Jersey, 1965).

${ }^{18} \mathrm{H}$. Brenner and M. E. O'Neill, Chem. Eng. Sci. 27, 1421 (1972).

${ }^{19}$ E. J. Hinch, J. Fluid Mech. 54, 423 (1972).

${ }^{20} \mathrm{~J}$. M. Rallison, J. Fluid Mech. 88, 529 (1978).

${ }^{21}$ D. J. Evans, Mol. Phys. 37, 1745 (1979).

${ }^{22}$ R. W. O'Brien, J. Fluid Mech. 19, 17 (1979).

${ }^{23}$ W. R. Dean and M. E. O'Neill, Mathematika 10, 13 (1963).

${ }^{24}$ A. J. Goldman, R. G. Cox, and H. Brenner, Chem. Eng. Sci. 21, 1151 (1966).

${ }^{25}$ S. Wakiya, J. Phys. Soc. Jpn. 22, 1101 (1967).

${ }^{26}$ M. H. Davis, Chem. Eng. Sci. 24, 1769 (1969).

${ }^{27}$ C. J. Lin, K. J. Lee, and N. F. Sather, J. Fluid Mech. 43, 35 (1970).

${ }^{28}$ M. E. O'Neill and S. R. Majundar, Z. Angew. Math. Phys. 21, 164 (1970).

${ }^{29}$ M. H. Davis, NCAR-TN/STR-64, National Center for Atmospheric Research, Boulder, Colorado (1971).

${ }^{30}$ G. K. Batchelor and J. T. Green, J. Fluid Mech. 56, 375 (1972).

${ }^{31}$ A. Nir and A. Acrivos, J. Fluid Mech. 59, 209 (1973).

${ }^{32}$ E. Wachholder and N. F. Sather, J. Fluid Mech. 65, 417 (1974).

${ }^{33}$ R. G. Cox, Int. J. Multiphase Flow 1, 343 (1974).

${ }^{34}$ P. A. Arp and S. G. Mason, J. Colloid Interface Sci. 61, 21 (1977).

${ }^{35}$ P. M. Adler, J. Colloid Interface Sci. 84, 461 (1981).

${ }^{36}$ D. J. Jeffrey and Y. Onishi, J. Fluid Mech. (to be published).

${ }^{37}$ There is no proof that it is impossible for particles to overlap if only pairwise interactions are considered. Indeed, there may be special configurations which would instantaneously lead to relative velocities which do not vanish as the separation distances approach their minimum value. In a flowing suspension, however, at a short time $\Delta t$ later this special configuration would be lost, and experience has indicated the particles do not overlap.

${ }^{38}$ E. G. Vervey and J. Th. G. Overbeek, Theory of the Stability of Lyophobic Colloids (Elsevier, Amsterdam, 1948).

${ }^{39} \mathrm{~K}$. Takamura, H. L. Goldsmith, and S. G. Mason, J. Colloid Interface Sci. 82, $175(1981)$.

${ }^{40}$ In the notation of Ref. 34, $\Phi_{\mathrm{rad}}=(1 / \pi)(f+2 g)$, where $f$ and $g$ are elements of the shear resistance matrix $\Phi$.

${ }^{41}$ R. L. Hoffman, Trans. Soc. Rheol. 16, 155 (1972).

${ }^{42}$ P. G. deGennes, J. Phys. 40, 783 (1979). 